\title{
Constraining Binary Evolution with Gravitational Wave Measurements of Chirp Masses
}

\author{
Tomasz Bulik* and Krzysztof Belczyński ${ }^{\dagger}$ \\ *Nicolaus Copernicus Astronomical Center, Bartycka 18, 00716 Warsaw, Poland \\ ${ }^{\dagger}$ Northwestern University, Dept. of Physics \& Astronomy, 2145 Sheridan Rd., Evanston, IL 60208, \\ USA
}

\begin{abstract}
Using the StarTrack binary population synthesis code we investigate the properties of population of compact object binaries. Taking into account the selection effects we calculate the expected properties of the observed binaries. We analyze possible constraints on the stellar evolution models and find that an observed sample of about one hundred mergers will yield strong constraints on the binary evolution scenarios.
\end{abstract}

\section{INTRODUCTION}

During this meeting we have learned about the great progress in gravitational wave astronomy. LIGO has finished its first two data runs and the data analysis is being done. The sensitivity of this instrument is improving steadily. The volume of space in which LIGO is sensitive to sources of gravitational wave radiation is increasing. While the main challenge is still detection of gravitational waves to prove directly their existence, another set of question arises: once we see sources of gravitational waves what astrophysical significance shall they have? Can any astrophysical problems be solved with gravitational wave astronomy? In this paper we concentrate on the most promising sources of high frequency gravitational waves (at least in the opinion of the authors), i.e. on coalescences of compact object binaries. We know that such binaries exist and that all the observations in the electromagnetic domain are consistent with emission of gravitational waves by them. We also know that these objects will coalesce.

So far most of the work on such binaries in the context of gravitational wave observations has concentrated on calculating the expected rates for the interferometric detectors. This problem has been approached in two ways. The first approach was based upon studying and analyzing the known compact object binaries, and then considering selection effects to estimate the properties of the entire population of such sources to finally obtain the coalescence rate. The drawback of this approach is the small number statistics, or even zero object statistics, in the case of black hole neutron star or double black hole binaries. Moreover, the results have also suffered from the uncertainty in estimates of the selection effects [1, 2]. A second approach is based on studying the stellar evolution processes and detailed analysis of the formation paths of double compact object binaries [3, 4, 5, 6, 7, 8]. The main problem associated with these calculations is lack 
TABLE 1. Description of different population synthesis models used here.

\begin{tabular}{ll}
\hline Model & Description \\
\hline A & standard model described in Belczynski, Kalogera, Bulik (2002), but with \\
& $T_{\text {Hubble }}=15$ Gyrs \\
B1,7,11 & zero kicks, single Maxwellian with $\sigma=50,500, \mathrm{~km} \mathrm{~s}^{-1}$, \\
B13 & Paczynski (1990) kick with $V_{k}=600 \mathrm{~km} \mathrm{~s}^{-1}$ \\
C & no hyper-critical accretion onto NS/BH in CEs \\
E1-3 & $\alpha_{\text {CE }} \times \lambda=0.1,0.5,2$ \\
F1-2 & mass fraction accreted: $\mathrm{f}_{\mathrm{a}}=0.1,1$ \\
$\mathrm{G} 1-2$ & wind changed by $f_{\text {wind }}=0.5,2$ \\
$\mathrm{~J}$ & primary mass: $\propto M_{1}^{-2.35}$ \\
L1-2 & angular momentum of material lost in $\mathrm{MT}: j=0.5,2.0$ \\
M1-2 & initial mass ratio distribution: $\Phi(q) \propto q^{-2.7}, q^{3}$ \\
$\mathrm{O}$ & partial fall back for $5.0<M_{\mathrm{CO}}<14.0 M_{\odot}$ \\
$\mathrm{S}$ & all systems formed in circular orbits \\
Z1-2 & metallicity: $Z=0.01$, and $Z=0.0001$
\end{tabular}

of detailed knowledge of the physics of several important stages in the stellar evolution. The results depend strongly on a particular parametrization of such stages. For example the formation rates of double neutron star binaries strongly depend on the distribution of kick velocities newly nascent neutron stars receive in supernova explosions.

We have studied a different aspect of observations of gravitational waves from coalescing binaries [9] - a measurement of the chirp mass. This paper is an expansion of the already published results. Previously [9] we only considered the case of Euclidean space with constant star formation rate and here we present a consideration of cosmological effects. In section 2 we present the stellar population model, section 3 contains estimates of the constraints from chirp mass measurements, and we finish with conclusions in section 4 .

\section{CALCULATIONS}

We use the StarTrack binary population synthesis code [10], to analyze formation channels and properties of compact object binaries. Within the code the single stellar evolution is described using a set of analytical formulae [11]. We include the description of the main sequence stars, evolution on the Hertzsprung gap, the red giant branch, core helium burning phase, and the asymptotic giant branch. Moreover, we include a detailed description of helium stars on their main sequence and giant branch. The binary evolution description includes such processes as orbital changes due to wind mass loss, tidal interaction, and magnetic breaking. We allow for different models of mass transfers: conservative, quasi dynamic, common envelope evolution, and we investigate possibility of hyper accretion onto compact objects. Description of the supernovae explosions include the consideration of various kick velocity distributions and results of numerical modeling of the formation of compact objects [12]. We assume that the distributions of the initial parameters are independent: the mass of the primary is drawn from a $\propto M^{-2.7}$ distribution [13], the mass ratio comes from a flat distribution, the distribution of ec- 


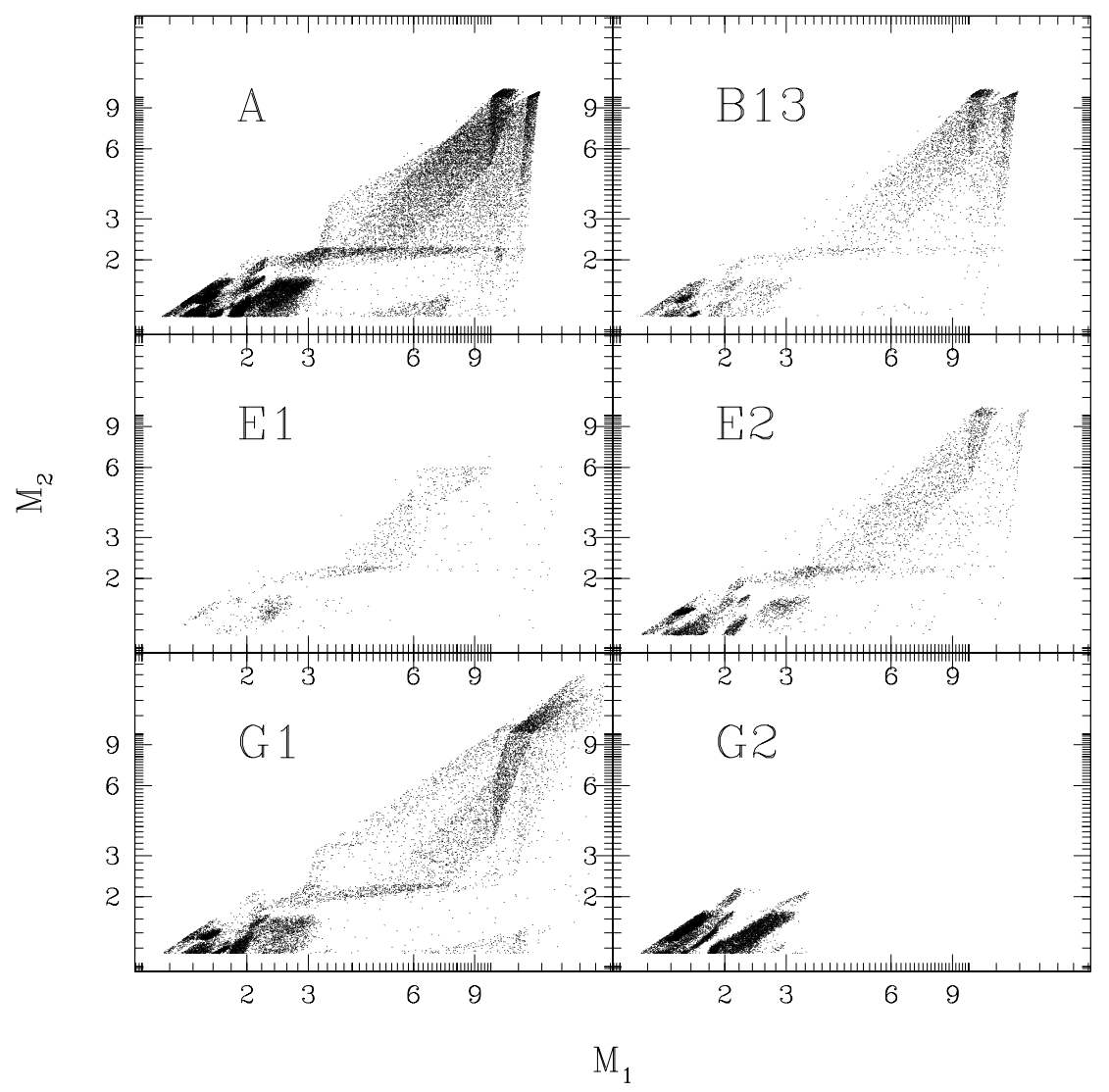

FIGURE 1. Tye distributions of masses of the population of compact object binaries in different models of stellar evolution, for description see Table 1.

centricity is $\propto e$, and the orbital separation distribution is flat in $\log a$. In order to test the sensitivity of the results we run the code varying several parameters. We denote our standard set of parameters as model A. Descriptions of other models used in this paper is listed in Table 1.

A typical calculation involves a few million initial binaries and results in several thousand compact object binaries. For each binary we note the masses of the individual objects and the orbital parameters. The distribution of the masses of the compact objects appears to be a very sensitive function of the model of stellar evolution. We present a few representative examples of such distributions in Figure 1 The models presented are model A, model B13 with a different kick velocity distribution, models E1 and E3 where we vary the efficiency of common envelope evolution and models G1 and G2 with decreased and increased stellar winds, respectively. The maximum masses of the compact object binaries vary from one model to another. In model G1 with decreased stellar winds there is a large number of double black hole binaries with masses up to $20 M_{\odot}$ which are absent in other models. In model E1 there are very little high mass ratio binaries, and the number of light (double neutron star) binaries is small compared 


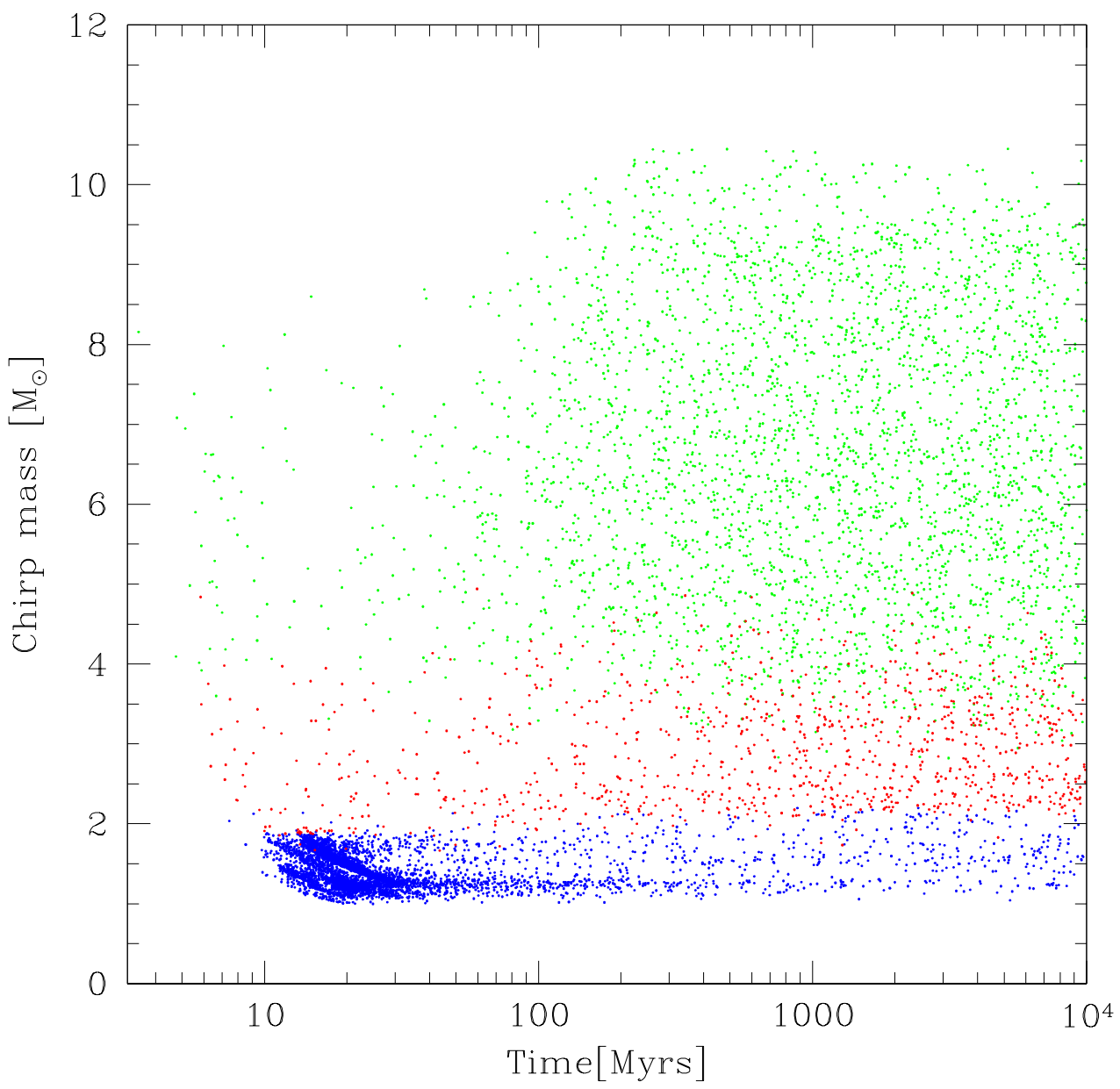

FIGURE 2. Population of the double compact objects within the framework of model A plotted in the space spanned by the chirp mass, and lifetimes counted from the formation of the system on zero age main sequence to the merger due to gravitational wave emission. The cutoff on the left side is due to nuclear evolution time.

to double black hole ones. Each model leads to a different pattern in the mass distribution which will affect predictions for the gravitational wave observations.

A fundamental quantity considered in calculating gravitational waveforms for coalescing binaries is the chirp mass $\mathscr{M}=\left(m_{1} m_{2}\right)^{0.6}\left(m_{1}+m_{2}\right)^{-0.2}$, where $m_{1}$ and $m_{2}$ are the individual masses of the binary components. In Figure 2 we plot the population of binaries from model $\mathrm{A}$ in the space spanned by the chirp mass and the binary lifetime 
$t_{\text {life }}$, defined as the duration from the formation of a system on zero age main sequence until its merger due to gravitational wave emission. A clear tendency is visible in the graph. The low chirp mass binaries, tend to merge predominantly on a short timescale, on the order of a few tens of million years. The high chirp mass binaries have longer lifetimes: a majority of them lives for a few Gyrs. This leads to an interesting conclusion: the currently merging double neutron stars originate in recent star formation episodes, while the the progenitors of currently merging double black holes date to a few billion years ago. Black hole neutron star binaries have chirp masses between 2 and $4 M_{\odot}$, and the distributions of their lifetimes is practically flat between a few tens of million years and the Hubble time.

\section{GRAVITATIONAL WAVE OBSERVATIONS}

We consider here detection of compact object coalescence in the inspiral phase and neglect the merger and ringdown signal. The signal to noise ratio from an inspiraling binary is proportional to $\propto[(1+z) \mathscr{M}]^{5 / 6} / d[15,16,17]$, where $d$ is the luminosity distance to the source, and $z$ is the redshift. For the stellar mass sources with chirp masses below $20 M_{\odot}$ the signal to noise from the merger and ringdown phases is weaker than the inspiral signal [17]. For a given, assumed signal to noise value required for a detection of a gravitational wave sources one can calculate the sampling distance (or redshift), i.e. a distance up to which such source should be detectable. The rate with which a detector will see mergers of a compact object binaries consisting of two masses $M_{1}$ and $M_{2}$ with a lifetimes $t_{\text {life }}$ is

$$
\frac{d R}{d M_{1} M_{2}}=\int_{0}^{z\left(M_{1} M_{2}\right)} \frac{d V}{d z} \frac{f_{M_{1} M_{2}} S F R\left(z+d z\left(t_{\text {life }}\right)\right)}{1+z} d z,
$$

where $\operatorname{SFR}(z)$ is the star formation rate function, $d z\left(t_{\text {life }}\right)$ is the retardation due to the lifetime of the binary, and $f_{M_{1} M_{2}}$ is the probability of forming a binary with the masses $M_{1}$ and $M_{2}$, while $z\left(M_{1} M_{2}\right)$ is the sampling redshift out to which the detector is sensitive to such mergers. The population synthesis code provides an estimate of the function $f_{M_{1} M_{2}}$, and gives us lifetimes of the binaries. In the Euclidean case with constant star formation rate history this integration is elementary and yields [9]:

$$
\frac{d R}{d M_{1} M_{2}} \propto \mathscr{M}^{5 / 2}
$$

In order to calculate the actual rate observed by a given detector one needs a good estimate of the function $f_{M_{1} M_{2}}$. A population synthesis code provides an estimate of this function, i.e. the number of such compact object binaries produced out of the initial population considered by the code. One has to bear in mind that we do not simulate the entire stellar population, and a number of uncertain factors enters a calculation of the rate. First, we assume a binary fraction of 0.5 , yet this number could be different for real stellar population. Second the compact object binaries originate in stars at the high mass end of the initial mass function. We assume that the the initial mass function is 
a power law, but its shape is not well known especially at the high end. The number of the compact object binaries depends strongly on the number of their progenitors. Only a small fraction of the total mass of the stars formed lies in the high mass stars. An additional uncertainty may come from different low mass cutoffs of the initial mass function which affects the total number of stars considered. The uncertainty in the rate from these factors can easily be larger than plus or minus a factor of ten. Additionally since the evolutionary times are important for the compact object binaries one should know the star formation history in our local group over that last few billion years. Apart from that, different models of stellar population lead different estimates of $f_{M_{1} M_{2}}$ [10]. The difference between the models when all other parameters are kept constant is up to factor of a hundred between the most pessimistic and most optimistic models. Thus given an observed rate will very hardly lead to strong constraints on the stellar evolution model. Different models do predict different rates when all the normalization parameters are kept constant. However the uncertainty of the rates calculated within each model are large enough that imposing constraints based solely on the rates will be a difficult, if not impossible task. Moreover, the gravitational wave interferometers exhibit non-stationary noise, which hampers estimates of the times space volume sampled by a given detector.

Fortunately, future detections of gravitational waves from coalescing binaries will not produce the rates alone. Each detection will be accompanied by a measurement of the chirp mass of the coalescing binary, and with good templates and post Newtonian analysis it should be possible to measure individual masses, and perhaps even the spins. For the sake of this paper we will only consider measurements of chirp masses. A galactic population of compact object binaries is heavily dominated by small chirp mass objects, see Figure 2 However, the detectability weighted distribution will be different. This is due to the fact the large chirp mass objects are visible to a larger distance. Here we assume that the star formation rate history is given as in Figure 5 of [18], i.e. it increases with redshift by a factor of more than ten up to $z=2$ and then remains flat until $z=30$. We also assume a cosmological model with the Hubble constant $H=65 \mathrm{~km} \mathrm{~s}^{-1} \mathrm{Mpc}^{-1}$, with $\Omega_{m}=0.3$ and $\Omega_{\Lambda}=0.7$, and the model detector used for the purpose of this calculation is sensitive $(S / N=8)$ to a merger of a $\mathscr{M}=1.2 M_{\odot}$ binary out to $125 \mathrm{Mpc}$. We present the differential distribution of the detectability weighted distribution of chirp masses in Figure 3. We should note that a distribution of chirp masses as a statistic is free from nearly all of the problems that the rate has suffered from. The distribution of chirp masses consists essentially of the ratios of one group of mergers to another. Therefore the absolute normalization that has been such a problem in applying the rate calculation cancels out. The distribution of Figure 3 peaks at the high chirp mass objects. This is because the volume in which compact object coalescences are detectable increases rapidly with the chirp mass, see eq. 2]

In the Euclidean space with constant star formation rate the distribution of observed chirp masses depends only on the assumed model of stellar evolution. In Figure 4 we present how the detectability weighted chirp mass distribution changes when the sensitivity of the detector is increased, by a factor of ten and hundred. The differences are small, the distribution are nearly the same when the sensitivity is increased by a factor of ten. The increase of the sensitivity by a next factor of ten increases the number of low chirp mass binaries in the observed sample. At this point the cosmological effects play a role. The sampling distance to the large chirp mass objects is large, however there 


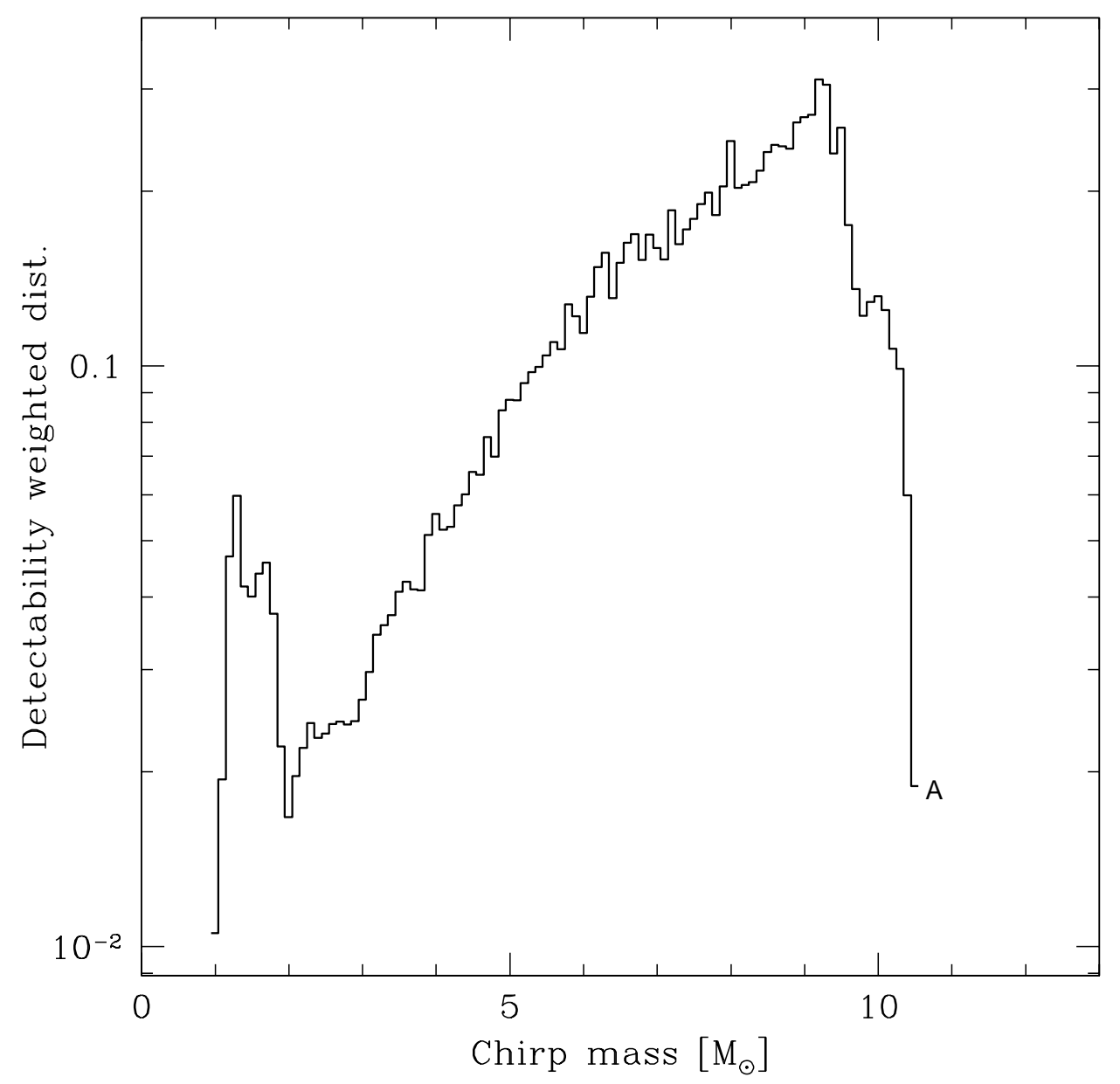

FIGURE 3. The detectability weighted differential distribution of the chirp mass within the model A. Double neutron star mergers constitute only less than a few percent of all the events.

is not much volume at high redshifts. This increase of sensitivity opens up space for more detections of low chirp mass binaries, but such detectors essentially see all double black hole mergers in the entire Universe so increasing the sensitivity does not increase the number of high chirp mass sources any more. A convenient method to compare two distributions is to use the Kolmogorov-Smirnov test. This test uses a maximum distance between the cumulative distributions $D$ to quantify the difference between them. The parameter $D$ between the solid and dotted line is very small - less then 0.01 , while between the solid and dashed lines it is $\approx 0.07$. In order to distinguish two distributions at a significance level of about $10^{-4}$ one needs to sample the distributions with $N=4 / D^{2}$ points.

In Figure 5 we present the cumulative distributions of detectability weighted chirp masses for the models listed in Table 1. For comparison we also show in each panel the distribution corresponding to our model A. The differences between the distributions are 


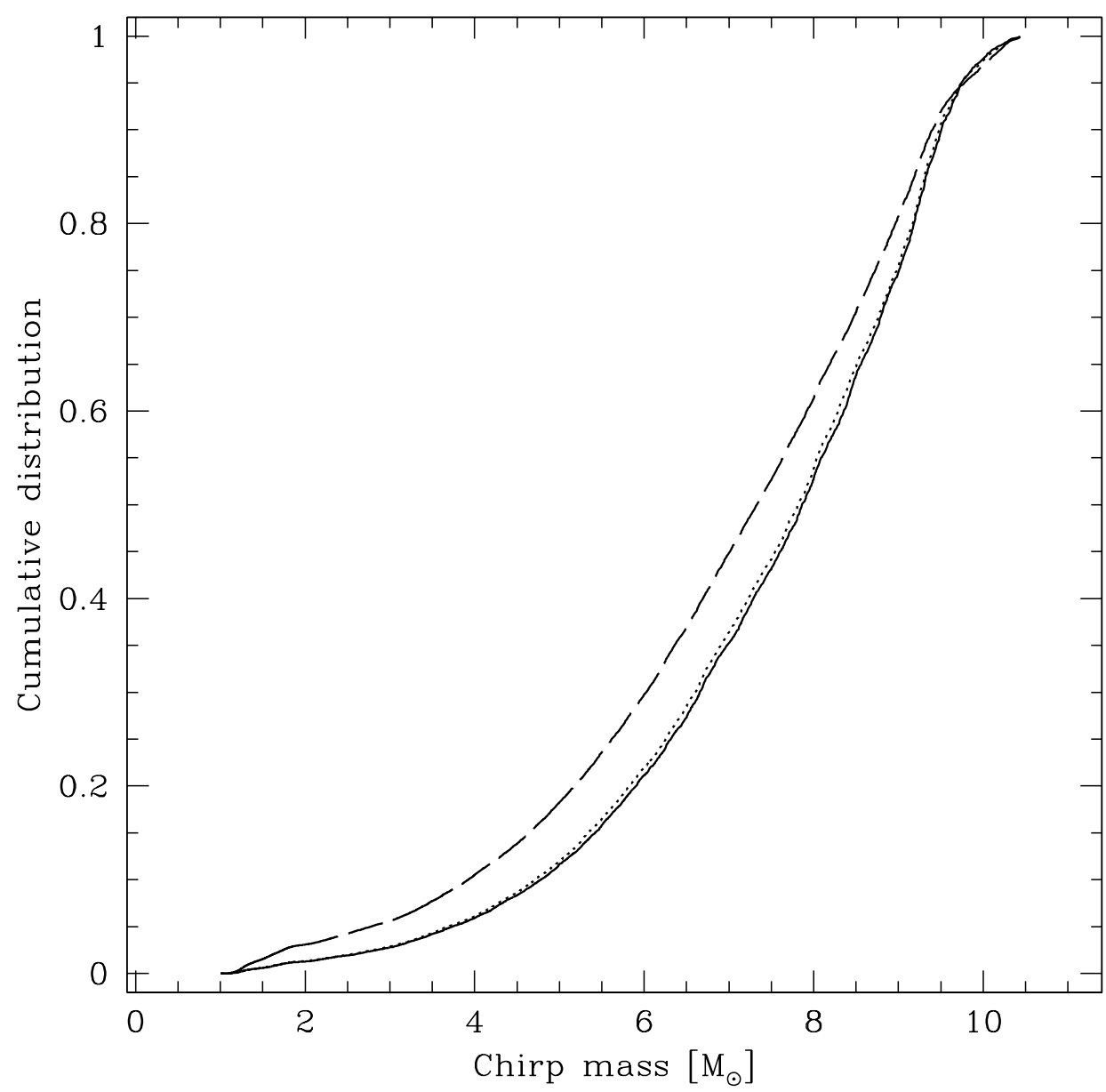

FIGURE 4. The cumulative distributions of detectability weighted chirp masses. the solid line corresponds to our fiducial model, while the dotted and dashed lines represent models where detectors are more sensitive by a factor of ten and hundred, respectively.

much larger than these in Figure 4 and for some models the parameter $D$ is larger than 0.5 ! This shows that a distribution of observed chirp masses carries a huge amount of information about the underlying stellar evolution model. It has been shown [9] that most of the stellar evolutionary parameters can be constrained with a sample of one hundred merger observations. Some parameters affect the observed distribution of chirp masses even stronger: several models can be ruled out with a sample of less than 20 measured chirp masses. Since the observed sample is dominated by the largest chirp mass objects the strongest constraints shall be imposed on these stellar evolutionary parameters that affect production of massive black holes. These are in particular the details of the core collapse of massive stars in supernovae explosions, and also the averaged winds of most massive stars that are the progenitors of highest mass black holes. It is important to repeat that a distribution of chirp masses is a statistic that is free of most of the systematic uncertainties, which constitute a problem, when one considers just the observed rate. 


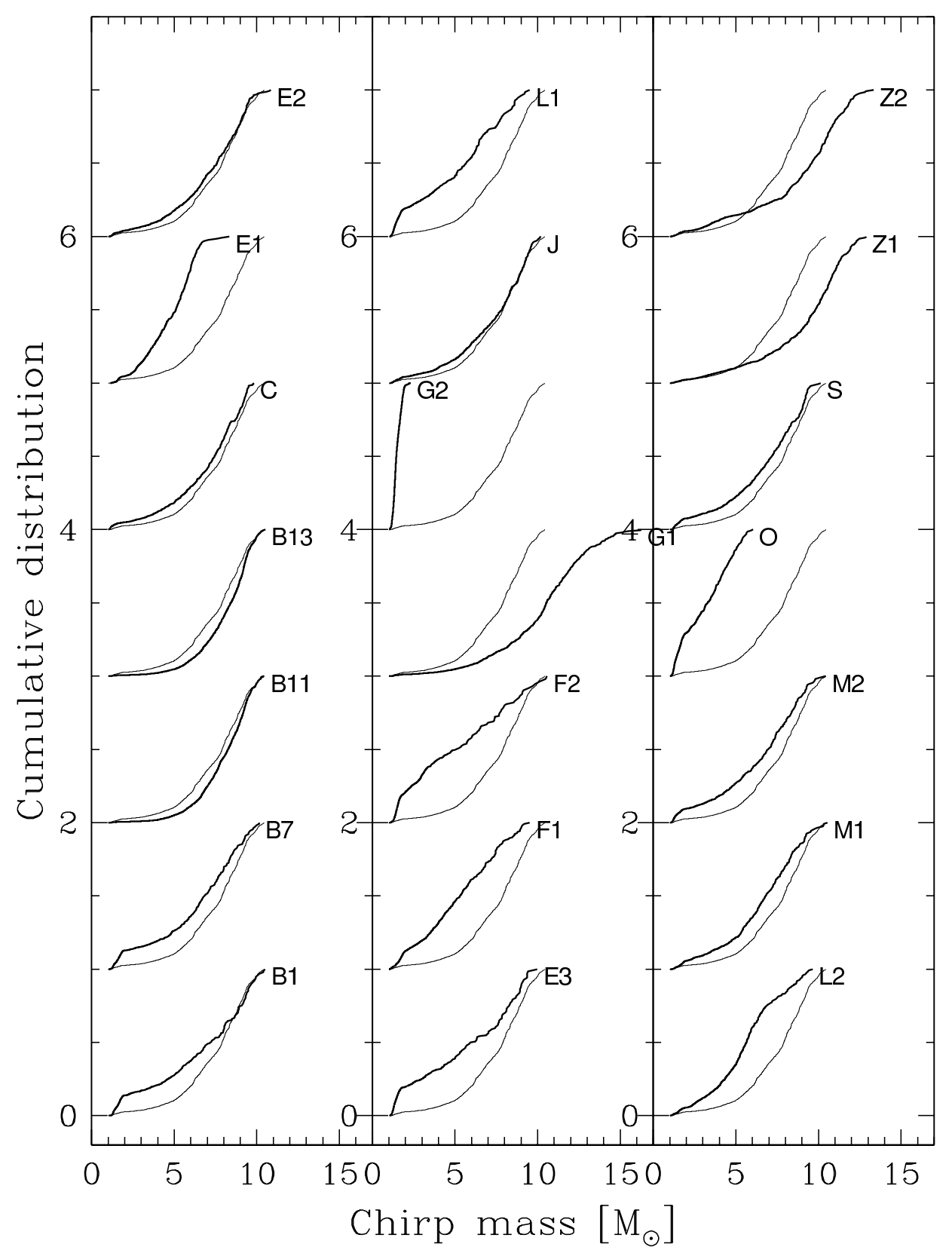

FIGURE 5. Cumulative distributions of detectability weighted chirp mass within the models listed in Table 1. For comparison we also show with thin lines the distribution of model A .

\section{CONCLUSIONS}

In this paper we presented the properties of the population of compact object binaries relevant for the gravitational wave merger calculations. The lifetimes of different types of binaries vary: the low mass binaries have shorter lifetimes than the heavy ones. The 
lifetimes of double black hole binaries are comparable to the Hubble time and therefore their progenitors can originate in the epochs when star formation rate was much higher than it is now. On the other hand the population of double neutron stars is short lived and it originates in recent star bursts.

We discuss two possible observational statistics that can be used to constrain stellar models: the observed rates, and the distribution of observed chirp masses. We argue that the rates alone shall impose very weak constraints on the stellar models, because of huge uncertainties in the models, as well as possible difficulties in dealing with non stationary noise in the detectors. The distribution of chirp masses is a statistics that is free from such uncertainties. Most probably even stronger bounds would be imposed if individual masses of coalescing objects are measured.

Finally we confirm the suggestion [19] that the observed sample is dominated by double black hole binaries. In all the models that we consider the observed sample is dominated by the highest chirp mass objects.

\section{ACKNOWLEDGMENTS}

This research was funded by the KBN grant 5P03D 01120 . TB thanks the organizers of the meeting for support.

\section{REFERENCES}

1. Narayan, R., Piran, T., and Shemi, A., Astroph. J. Lett., 379, L17-L20 (1991).

2. Kalogera, V., Narayan, R., Spergel, D. N., and Taylor, J. H., Astroph. J., 556, 340-356 (2001).

3. Lipunov, V. M., Postnov, K. A., and Prokhorov, M. E., M.N.R.A.S., 288, 245-259 (1997).

4. Fryer, C., Burrows, A., and Benz, W., Astroph. J., 496, 333-+ (1998).

5. Portegies Zwart, S. F., and Yungelson, L. R., Astronomy and Astrophysics, 332, 173-188 (1998).

6. Bethe, H. A., and Brown, G. E., Astroph. J., 506, 780-789 (1998).

7. Fryer, C. L., Woosley, S. E., and Hartmann, D. H., Astroph. J., 526, 152-177 (1999).

8. Bulik, T., Belczyński, K., and Zbijewski, W., M.N.R.A.S., 309, 629-635 (1999).

9. Bulik, T., and Belczyński, K., Astroph. J. Lett., 589, L37-L40 (2003).

10. Belczynski, K., Kalogera, V., and Bulik, T., Astroph. J., 572, 407-431 (2002).

11. Hurley, J. R., Pols, O. R., and Tout, C. A., M.N.R.A.S., 315, 543-569 (2000).

12. Fryer, C. L., Astroph. J., 522, 413-418 (1999).

13. Scalo, J. M., Fundamentals of Cosmic Physics, 11, 1-278 (1986).

14. Paczynski, B., Astroph. J., 348, 485-494 (1990).

15. Chernoff, D. F., and Finn, L. S., Astroph. J. Lett., 411, L5-L8 (1993).

16. Bonazzola, S., and Marck, J. A., Annu. Rev. Nucl. Part. Sci., 45, 655 (1994).

17. Flanagan, É. É., and Hughes, S. A., Phys. Rev. D, 57, 4535-4565 (1998).

18. Belczynski, K., Bulik, T., and Rudak, B., Astroph. J., 571, 394-412 (2002).

19. Lipunov, V. M., Postnov, K. A., and Prokhorov, M. E., New Astronomy, 2, 43-52 (1997). 